\title{
ABORTO Y PARTICIPACIÓN POLÍTICA EN LA ETIQUETA \#28S EN ARGENTINA
}

\section{ABORTION AND POLITICAL PARTICIPATION ON THE \#28S IN ARGENTINA}

\author{
MARINA ACOSTA
}

Author / Autora:

Marina Acosta

Universidad de Buenos Aires

Buenos Aires, Argentina

macosta@sociales.uba.ar

https://orcid.org/0000-0002-8270-4372

Submitted / Recibido: 05/03/2021

Accepted / Aceptado: 21/06/2021

To cite this article / Para citar este artículo: Acosta, M. (2022). Aborto y participación política en la etiqueta \#28S en Argentina. Feminismo/s, 39, 13-37. https://doi. org/10.14198/fem.2022.39.01

\section{Licence / Licencia:}

This work is licensed under a Creative Commons Attribution 4.0 International.

\section{(c) (i)}

(C) Marina Acosta

\section{Resumen}

El feminismo es uno de los movimientos sociales que más relevancia ha cobrado en el contexto mundial en los últimos tiempos. Con una larga historia de lucha, las mujeres son portadoras de demandas que se vertebran en disputas articuladas tanto en premisas de reconocimiento como de redistribución. La participación de muchas mujeres jóvenes en las movilizaciones del \#NiUnaMenos obligó al feminismo a apelar a otras formas de comunicación y servirse de las plataformas digitales para organizar la protesta social. Las redes sociales le han proporcionado al colectivo una plataforma para visibilizar sus reclamos y demandas globales. En el Día Internacional por el Aborto Seguro, la Campaña Nacional por el Derecho al Aborto legal, seguro y gratuito lanzó en Argentina la etiqueta \#28S con el fin de presionar a los tomadores de decisión en la discusión formal sobre el aborto. La etiqueta arropó los sentires y opiniones de muchos/as ciudadanos/as preocupados/as por la problemática. Como en general ocurre con el activism hashtag los/las usuarios/as crearon un conjunto de narrativas de petición, demandas y protestas. El objetivo de la investigación es analizar la conversación digital en el hashtag para dar cuenta de la dinámica comunicacional 
de los/las usuarios/as que participaron de ella, describir sus interacciones e identificar sus narrativas. Además de la clásica revisión bibliográfica, la propuesta cuali-cuantitativa del estudio es exploratoria y descriptiva. Se aplica el método cuantitativo del análisis de contenido y el método cualitativo del análisis documental. Dentro de los principales resultados destaca que las cuentas más activas pertenecen a usuarios/as con poca cantidad de seguidores que escriben a título personal, los líderes de opinión crean los contenidos alrededor de los cuales se produce la interacción y en sus publicaciones los/las usuarios/as retoman los encuadres activados por la Campaña en sus documentos.

Palabras clave: aborto; feminismo; redes sociales; Twitter, comunicación digital.

\begin{abstract}
Feminism is one of the social movements that has gained the most relevance in the world context in recent times. With a long history of struggle, women are bearers of demands that are structured in disputes articulated both on premises of recognition and redistribution. The participation of many young women in the \#NiUnaMenos mobilizations forced feminism to appeal to other forms of communication and use digital platforms to organize social protest. Social networks have provided the collective with a platform to make their global claims and demands visible. On the International Day for Safe Abortion, in Argentina, the National Campaign for the Right to Legal, Safe and Free Abortion launched the \#28S to put pressure on decision makers about the formal discussion on abortion. The objective of the research is to analyse the digital conversation that arose around the hashtag to observe the communicational dynamics of the users who participated in it, describe their interactions and identify their claims and narratives. In addition to the classic bibliographic review, the qualitative-quantitative proposal of the study is exploratory and descriptive. The quantitative method of content analysis and combined the qualitative method of documentary analysis. Among the main results, it stands out that the most active accounts belonged to users with a low number of followers who wrote on a personal basis, that those who produce the content around which users interact are opinion leaders and that in their publications, users returned the frames activated by the Campaign in their public documents.
\end{abstract}

Keywords: abortion; feminism; social media; Twitter; digital communication. 


\section{INTRODUCCIÓN}

La utilización de los recursos comunicacionales para visibilizar diversos tipos de demandas sociales ha sido abordada durante el siglo pasado en trabajos clásicos del campo de estudio de la acción colectiva que observaban las dinámicas de actuación de los movimientos sociales (della Porta, 2008; della Porta \& Diani, 2015; McAdam et al., 2005; Tarrow, 2005; Tilly, 2005). El desarrollo de las nuevas tecnologías de la información y las nuevas formas de autocomunicación de masas han amplificado las posibilidades de ejercicio de la acción colectiva y se han incorporado al escenario de la protesta y el activismo (Castells, 2009, 2013).

El feminismo es uno de los movimientos sociales que más relevancia ha cobrado en el contexto mundial en los últimos tiempos. Con una larga historia de lucha, las mujeres son portadoras de demandas que se vertebran en disputas articuladas tanto en premisas de reconocimiento como de redistribución (Fraser, 2008, 2012). Las redes sociales le han proporcionado al colectivo una plataforma para visibilizar sus reclamos y demandas globales (Arias Rodríguez, 2017; Nathanson \& Paz, 2019; Núñez Puente \& Fernández Romero, 2017; Sábada \& Barranquero, 2019; Treré, 2016).

Tal es el caso de lo que ocurrió en Argentina desde 2015 cuando el feminismo encontró en las redes sociales un nuevo aliado para agendar sus reivindicaciones. La irrupción del \#NiUnaMenos se convirtió en un parteaguas para el movimiento (Accossatto \& Sendra, 2018; Acosta, 2018b; Natalucci \& Rey, 2018; Rosales, 2018). La amplia tematización sobre la violencia de género que instalaron sus marchas masivas permitió, además, que la demanda por el aborto legal encontrara mayor receptividad en la agenda pública. Con ese escenario, en 2018, la Campaña Nacional por el Derecho al Aborto legal, seguro y gratuito (en adelante, la Campaña), que desde 2005 venía trabajando intensamente en la temática (Alcaraz, 2018; Ramírez Morales \& Felitti, 2020), emprendió una exitosa campaña de tecnopolítica (Toret, 2013, 2015). El corolario fue la sanción de la ley 27.610, a fines de 2020, que establece el acceso a la interrupción voluntaria del embarazo en todo el territorio nacional ${ }^{1}$.

1. Fuente: https://bit.ly/2ZsM4nh Consulta: enero de 2021.

Feminismo/s 39, January 2022, 13-37 
La participación de muchas mujeres jóvenes en las movilizaciones del \#NiUnaMenos (Elizalde y Mateo, 2018) obligó al feminismo a apelar a otras formas de comunicación y servirse de las plataformas digitales para organizar la protesta social. La Campaña utilizó a los medios sociales como recursos cívicos para el compromiso y la participación política (Dahlgren, 2018) y llevó adelante distintos tipos de intervenciones en el espacio público tradicional y una estrategia digital sólida en las redes sociales más utilizadas por los/las argentinos/as como Facebook, Twitter e Instagram (Acosta, 2018a; Acosta, 2020).

Tras la frustrada sanción de la normativa bajo la administración macrista (2015-2019), los esfuerzos para mantener el tema en la agenda se incrementaron. La llegada de la pandemia de la COVID-19 no fue impedimento para que en 2020 la Campaña lanzara la etiqueta \#28S ya con un nuevo gobierno de orientación centro-izquierda (Frente de Todos) que había prometido durante la campaña electoral presentar un proyecto que recogiera la demanda por la legalización del aborto. En el Día Internacional por el Aborto Seguro -28 de septiembre- se trataba de llamar la atención de los tomadores de decisión para que no retrasaran la discusión formal y reglamentaran un derecho históricamente postergado. La Campaña activaba, de esa manera, una nueva estrategia de actuación en el espacio público digital que buscaba aprovechar las oportunidades políticas, insistir en la creación de identidades colectivas, reivindicar la lucha y movilizar a aquellos/as que pedían por la norma (Tarrow, 2012).

La etiqueta arropó los sentires y opiniones de muchos/as ciudadanos/as preocupados/as por la problemática. Como en general ocurre con el activism hashtag (Clark, 2016; Konnelly, 2015; Yang, 2016), los usuarios/as crearon un conjunto de narrativas de petición, demandas y protestas. Así, el objetivo de la investigación es analizar la conversación digital activada en torno a la etiqueta \#28S para dar cuenta de la dinámica comunicacional de los/las usuarios/as que participaron de ella, describir sus interacciones e identificar sus reclamos. La propuesta cuali-cuantitativa del estudio es exploratoria y descriptiva. Se aplica, en este sentido, el método cuantitativo del análisis de contenido y el método cualitativo del análisis documental. 


\subsection{Contexto}

En 2005, un colectivo feminista inició la Campaña. Se recogía, así, una larga tradición de lucha por el derecho legal al aborto, hasta entonces una demanda largamente postergada para las mujeres y el conjunto de la ciudadanía. Entre sus principios fundantes destacan la libertad y autonomía de las mujeres para decidir (Gutiérrez, 2014). Su labor ha sido fundamental para posicionar la problemática del aborto en la esfera pública.

Durante 2018 la democracia argentina asistió al debate del aborto que hasta ese entonces había permanecido fuera de la agenda parlamentaria. El espacio público argentino experimentó un nuevo fenómeno de opinión pública discursiva (Sampedro \& Resina, 2010) que tuvo a las redes sociales como epicentro: el debate formal sobre el tema se dio en el Congreso de la Nación pero la autocomunicación de masas se convirtió en la protagonista de esa discusión histórica en tanto propició un intenso momento de democracia deliberativa (Habermas, 1998).

La expectativa ciudadana fue enorme pues, por primera vez, se trataría un proyecto presentado en conjunto por diputados oficialistas y opositores para despenalizar el aborto. Las redes sociales se transformaron en los lugares privilegiados donde se desarrolló el intercambio discursivo. Allí bajo la etiqueta \#AbortoLegalYa en Twitter y las páginas de Facebook e Instagram de la Campaña se organizó la estrategia tecnopolítica a favor de la interrupción voluntaria del embarazo.

El 14 de junio de ese año, después de casi veintitrés horas de debate, la Cámara baja aprobó (en general) el proyecto, con 129 votos a favor, 125 en contra y 1 abstención. Fue un final incierto dado que varios/as diputados/as no habían hecho público su voto. Tras pasar al Senado, el 9 de agosto, después de dieciséis horas de debate, una mayoría antiabortista le obturó el camino a la sanción de la ley con 38 votos en contra, 31 a favor y dos abstenciones.

La Campaña quiso reactivar el debate en 2019 pero sus esfuerzos fueron infructuosos. En un año de elecciones presidenciales, la problemática del aborto quedaba relegada de las preocupaciones de la agenda partidaria.

La coyuntura pandémica de 2020 representó una nueva oportunidad para visibilizar sus demandas. En un contexto de emergencia sanitaria, donde la sanidad pública se concentraba en atender los casos de COVID-19, 
se profundizaban los obstáculos en el acceso a la salud sexual y reproductiva. En noviembre de ese año, el Ejecutivo presentó un nuevo proyecto para legalizar el aborto y crear un programa para fortalecer la atención integral de la salud en la mujer durante el embarazo. La Campaña aprovechó la oportunidad para reforzar sus intervenciones y reclamos en el espacio público digital.

\subsection{Participación política}

La acción política puede ajustarse a formas convencionales (aceptadas y legitimadas por la comunidad porque coinciden con los valores dominantes) o no convencionales (formas de «hacer política» que entran en conflicto con los valores dominantes y suelen desarrollarse al margen de los cauces institucionales) (Tarrow, 2012; Tilly, 2010). En cualquier caso, la participación política nunca comienza como tabula rasa, al contrario está condicionada tanto por circunstancias externas como por los recursos con los que cuentan los/las ciudadanos/as (Dahlgren, 2018, p. 29).

La rapidez y ubicuidad de los nuevos canales de participación y expresión que ofrecen las plataformas digitales permiten la expansión espaciotemporal de la acción política, añadiéndole un componente de interactividad inmediata (Vallès \& Martí, 2016, pp. 332-339). Las formas de participación política cambian en la sociedad de la información y el conocimiento (Castells, 1998) y se materializan en otros espacios que exceden las estructuras institucionales formales. Los repertorios de acción colectiva se reinventan en las plataformas digitales (della Porta \& Diani, 2011; Tilly, 2000; 2005) y son utilizados por los actores sociales como campos de contienda para expresar lo que les sucede (Natalucci \& Rey, 2018).

En los picos de protesta, la acción colectiva encuentra en estas formas de participación política no convencional un partner fundamental en tanto en las redes sociales se crean mecanismos de coordinación claves para la movilización social (Gerbaudo, 2012; Resina de la Fuente, 2010) que complementan la acción política tradicional (Sola-Morales \& Sabariego, 2020). Internet facilita y actúa como soporte de la acción colectiva en términos de organización y movilización a la vez que produce nuevos modos de acción colectiva. En otras palabras, cumple una función facilitadora y creativa (VanLaer \& Van-Aelst, 2010). 
El ecosistema de medios conectivos representa una infraestructura dinámica capaz de influir en la cultura (van Dijck, 2016) y en la política (Castells, 2009; Ceron et al., 2013). De esta manera, los actores sociales interesados en visibilizar sus demandas deben desarrollar un sistema de comunicación eficaz basado en la difusión de información que vaya en dos direcciones: a los tomadores de decisión y a otros actores sociales que puedan convertirse en aliados. En el caso del \#28S la acción política no convencional tomó la forma de una acción de protesta para manifestar un descontento (Norris, 2002). La Campaña impulsó la etiqueta precisamente para que, a través de la viralización de los mensajes y el consecuente acto de habla colectivo, el sistema político se sintiera presionado. El hashtag, además, actuó como dispositivo que facilitó la identidad colectiva de los/las usuarios/as (Konnelly, 2015).

\subsection{Objetivos, preguntas e hipótesis}

En las últimas décadas, en América Latina hubo avances respecto de derechos civiles y sexuales que redundaron en mejoras para la vida de las mujeres y las niñas. Los movimientos feministas y de mujeres tuvieron una activa y vital participación para impulsar cambios que hoy se traducen, principalmente, en marcos normativos.

Como dijéramos ut supra, en la problemática relacionada con el aborto, el feminismo argentino ha realizado un intenso y continuo trabajo en pos de incluirlo en la agenda pública. Para ello, han recurrido a nuevas formas de acción política; por caso, apelando también a las nuevas tecnologías de la información y la comunicación que les permitieron amplificar la visibilidad de sus reclamos y convocar al mismo tiempo a las nuevas generaciones de mujeres que no habían sido hasta entonces interpeladas. El hashtivismo opera, en efecto, como un nuevo recurso para la movilización (Clark, 2016; Georgakopoulou, 2017; Yang, 2016).

En tanto la proximidad en el espacio describe cierta afinidad, la interacción reiterada y sostenida define una comunidad (Calvo y Aruguete, 2020). Los/las usuarios/as que se vinculan en las plataformas digitales pueden discutir, apoyarse, coincidir o entrar en desacuerdo. Las comunidades virtuales constituyen un conjunto de usuarios/as cuyos miembros interactúan entre sí e independientemente de sus posturas frente a los temas activan una relación. 
La conversación digital que se genera en las redes sociales resulta, entonces, de las interacciones dialógicas (tuits, retuits, favoritos, respuestas, menciones) que se activan alrededor de las etiquetas (Larrondo et al., 2019, p. 209).

Así, el objetivo general de la investigación es analizar la conversación digital de los/las usuarios/as de la etiqueta \#28S. Específicamente, el estudio se propone: 1) caracterizar las cuentas más activas en la etiqueta; 2) identificar los/las usuarios/as que generaron más interacciones; 3) registrar las palabras usadas con más frecuencia en las publicaciones.

De estos objetivos se derivan las siguientes preguntas de investigación (Q):

- Q1: ¿qué tipos de usuarios/as utilizan la etiqueta?, ¿tienen muchos o pocos seguidores?;

- Q2: ¿quiénes producen los contenidos que generan engagement con los/las usuarios/as?, ¿cuáles son los recursos comunicacionales que utilizan en sus publicaciones?;

- Q3: ¿qué tipo de narrativas se activan?, ¿se retoman las consignas impulsadas por la Campaña?

Las hipótesis de trabajo son:

- H1: En la etiqueta predominan usuarios/as que participan a título personal. En general, son cuentas que tienen pocos seguidores.

- H2: La conversación digital es guiada por las cuentas de los líderes de opinión que son las que producen mayores niveles de interacción.

- H3: Las narrativas que producen los/las usuarios/as están alineadas a las consignas de la Campaña.

\section{METODOLOGÍA}

\subsection{Diseño metodológico}

Además de la clásica revisión bibliográfica, la propuesta cuali-cuantitativa de esta investigación es exploratoria y descriptiva (Hernández Sampieri et al., 1991). A continuación, detallamos el protocolo de análisis.

Para enriquecer el análisis y evitar ciertos sesgos que pueden derivarse del proceso de investigación con una única metodología, el estudio adopta el enfoque integrado multimodal (Cresswell, 2015; Hernández Sampieri et al., 
1991). Aplicamos el método cuantitativo del análisis de contenido (Bardin, 1986) y lo combinamos con el método cualitativo del análisis documental (Valles, 2000). De esta forma, obtenemos una mayor variedad de perspectivas del problema.

Twitter ofrece una enorme cantidad de datos y metadatos por lo que se ha recurrido al social media mining que nos permite no sólo capturar y analizar los datos generados por los/las usuarios/as en sus interacciones (Zafarani et al., 2014) sino además comprender los flujos de información y obtener conocimientos profundos sobre la estructura de la red social que es objeto de estudio (Gundecha \& Liu, 2014).

La captura y manipulación de los datos de Twitter fue realizada en el entorno de programación de RStudio. Con el paquete TwitteR recuperamos 8.200 tuits originales (se excluyeron retuits) publicados en el hashtag \#28S durante el 28 de septiembre de 2020. Las unidades de análisis (publicaciones) fueron tabuladas en un data.frame compuesto de variables categóricas y numéricas.

Durante la semana previa al \#28S, se ha observado directamente el portal de la Campaña (www.abortolegal.com.ar) donde el equipo de comunicación digital ha subido documentos propios relacionados con las distintas actividades que propuso en el marco del \#28S. Estos documentos se han procesado en el programa ATLAS.ti de análisis cualitativo asistido por computadora (CAQDAS). Esta herramienta nos ha permitido clasificar datos y buscar códigos de patrones que se integraron en una misma unidad hermenéutica (Hwang, 2008).

Por tanto, el corpus de análisis de la investigación se compone de los tuits publicados en el hashtag de Twitter $(n=8.200)$ y de los documentos subidos a la página de Internet de la Campaña $(n=2)$.

\subsection{Operacionalización de variables cuantitativas}

Para realizar la operacionalización de las variables cuantitativas se ha seguido el esquema propuesto por Padua (2000): definición conceptual, dimensiones que la componen y elaboración de indicadores para iniciar la contrastación empírica. 
Para determinar la naturaleza de la persona que administra un perfil, las cuentas pueden categorizarse en personales, institucionales y personajes surgidos en la red (fakes). Las personales son aquellas que crean personas físicas, con datos identificativos asociables directamente a su gestor. Las institucionales pretenden representar a un colectivo empresarial, una asociación u organización de cualquier índole, sin importar si su fin es social, comercial o de otro tipo. Están asociadas a valores de marca. Finalmente, las cuentas paródicas se valen de las posibilidades que brinda el anonimato en Internet para conformar una suerte de personaje que no se asocia a la persona de su creador sino a una especie de marca personal (Deltell et al., 2013).

Si se sigue a la UK's Advertising Standards Authority, se habla de cuentas con muchos seguidores cuando el usuario supera los 30.000; por el contrario, aquellos que tienen menos de esa cantidad son usuarios con pocos seguidores ${ }^{2}$.

El alcance de una publicación se mide por las interacciones que produce (retuits y likes). Para medir el impacto de los tuits, recurrimos al concepto de propagación; el retuit (RT) es una acción que se utiliza en la retransmisión de tuits de otros/otras usuarios/as, de manera que el mensaje aparezca multiplicado y asociado al autor/a original. Dar retuit o likear a un/una usuario/a implica apoyar lo que éste/ésta publica (Congosto et al., 2013).

En las publicaciones dentro de una etiqueta, además, los/las usuarios/ as exponen sus ideas. De ellas se pueden inferir las narrativas. A través del análisis de la frecuencia en el uso de palabras se alcanza un alto nivel de descripción de esos discursos (Silge \& Robinson, 2017). La minería de texto nos ayuda a descubrir conocimientos en corpus textuales a partir de la identificación y exploración de ciertos patrones de la red de comunicación que se activa entre los/las usuarios/as que participan de una conversación digital (Pérez Guadarramas et al., 2017). La Tabla 1 muestra las variables de análisis.

2. Fuente: https://bit.ly/3dtsFKD Consulta: junio de 2021. 
Tabla 1. Operacionalización de variables

\begin{tabular}{|c|c|c|}
\hline Variable & Dimensiones e Indicadores & Referencia \\
\hline \multirow{4}{*}{ Usuarios/as } & Tipos & \multirow[b]{2}{*}{ Delltel et al. (2013) } \\
\hline & $\begin{array}{c}\text { Personal } \\
\text { Institucional } \\
\text { Personaje (Fake) }\end{array}$ & \\
\hline & Cantidad de seguidores & \multirow[b]{2}{*}{ UK's Advertising Standards Authority } \\
\hline & $\begin{array}{c}\text { Muchos }>30.000 \\
\text { Pocos }<30.000\end{array}$ & \\
\hline \multirow[b]{2}{*}{ Interacción } & Acción & \multirow[b]{2}{*}{ Glosario de Twitter } \\
\hline & $\begin{array}{c}\text { Retuits } \\
\text { Favorito (Me gusta) }\end{array}$ & \\
\hline \multirow[b]{2}{*}{ Opinión } & Tópicos & \multirow[b]{2}{*}{ Silge y Robinson (2017) } \\
\hline & Frecuencia & \\
\hline
\end{tabular}

Fuente: elaboración propia.

\section{ANÁLISIS Y RESULTADOS}

Los 8.200 tuits de la etiqueta \#28S fueron publicados por 5.467 usuarios/as. Las reacciones contabilizaron un total de 165.989 que se distribuyeron de la siguiente manera: a) 39.448 retuits con una media de 4.81 por usuario/a; b) 126.541 favoritos con una media de 15.43 por cuenta. La primera observación que hacemos, entonces, es que el nivel de interacción de los/las usuarios/as fue alto. Esta afirmación se corresponde con el hecho de que durante buena parte de la jornada la etiqueta fue tendencia en Twitter Argentina ${ }^{3}$.

La estrategia de la Campaña de espolear un conjunto de etiquetas auxiliares al \#28S también tuvo buenos resultados. En sus tuits, los usuarios/as apelaron a nuevos hashtags como \#AbortoLegal2020 ( $n=5.646)$ y \#EsUrgente

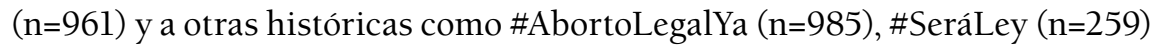
y \#QueSeaLey $(n=221)$, utilizadas en otros momentos álgidos del proceso de discusión pública. En cualquier caso, lo que aquí se advierte es que todas estas etiquetas crearon una relación social de afinidad entre los/las usuarios/ as (Zappavigna, 2015). Valga también aclarar que todas se encuadran en el tipo de «causa» (cause hashtag) en tanto buscan crear conciencia o reunir

3. Fuente: http://bit.ly/2ZGKkqV Consulta: enero de 2021.

Feminismo/s 39, January 2022, 13-37 
apoyo a una problemática social (Konnelly, 2015) como, en este caso, el aborto.

\subsection{Usuarios/as}

El movimiento feminista argentino ha conseguido constituir a lo largo de las décadas un contrapúblico subalterno que se desarrolló en un terreno discursivo paralelo en el cual hizo circular sus demandas. Su gran mérito fue haber logrado instalar el debate sobre el aborto más allá de ese contrapúblico (Lucaccini et al., 2019). Las redes sociales fueron grandes aliadas para que en ellas también se configurara un nuevo espacio que le permitiera a muchos/as ciudadanos/as apoyar ese discurso. Los/las usuarios/as que se expresan en las plataformas digitales sobre el tema suelen hacerlo a modo individual; es decir, sin necesidad de pertenecer orgánicamente a una institución o colectivo.

Las diez cuentas con más actividad dentro de la etiqueta pertenecen a usuarios/as con poca cantidad de seguidores, a excepción de la cuenta de la Campaña (@CampAbortoLegal) que fue por otra parte la que más veces realizó publicaciones, tal como se advierte en la Tabla 2.

Tabla 2. Usuarios/as con más actividad

\begin{tabular}{|c|c|c|c|}
\hline \multicolumn{4}{|c|}{ Tabla 2. Usuarios/as más activos/as } \\
\hline @CampAbortoLegal & Publicaciones & \multicolumn{1}{|c|}{ Seguidores } & Tipo de cuenta \\
@campaniabortoLP & 43 & 147.200 & Institucional \\
@Damiani5Claudia & 37 & 6.828 & Institucional \\
@delfuego__ & 32 & 43 & Personal \\
@PatoBQ_- & 29 & 133 & Personal \\
@RoFerrerllustra & 22 & 1.660 & Personal \\
@bicho_vazquez & 22 & 16.500 & Personal \\
@aleullua & 21 & 1.275 & Personal \\
@CampAbortoCABA & 19 & 3.938 & Personal \\
@RosaFig65873444 & 19 & 6.339 & Institucional \\
\hline
\end{tabular}

Fuente: elaboración propia.

Las cuentas corresponden mayormente a usuarios/as que escriben a título personal. Este tipo de usuarios/as son los que crearon una fuerte 
comunidad que mantuvo activa la dinámica conversacional. Gracias a estas cuentas las publicaciones de los/las usuarios/as con más seguidores alcanzaron mayor visibilidad.

La Campaña (@CampAbortoLegal) y sus diferentes capítulos en el país (@campaniabortoLP, @CampAbortoCABA) sumaron 99 publicaciones. Sus actividades guían la dinámica de participación de los/las usuarios/as a partir del valor del contenido de sus publicaciones. Si bien se han analizado los tipos de posteos que realizaron, la estrategia comunicacional que las caracteriza es mantener activo el debate a partir de la generación de contenidos. Además, a través del flujo constante de comunicación con los seguidores buscaron construir comunidad (Gong, 2014).

\subsection{Interacción}

Las plataformas digitales facilitan la interacción y la distribución de contenidos y experiencias de los/las usuarios/as. Ofrecen, en definitiva, nuevas potencialidades que pueden derivar en nuevas formas de relación social (Caro Castaño, 2015). Por eso resulta importante reparar en los tipos de relaciones que se producen, por caso, entre las cuentas que participan de una etiqueta y observar particularmente quiénes son los perfiles que definen los contenidos alrededor de los cuales interactúan los/las usuarios/as.

Nueve de las diez cuentas que han recibido más interacciones (favoritos y retuits) poseen muchos seguidores (> 30.000). Quienes producen, entonces, los contenidos alrededor de los cuales los/las usuarios/as interactúan son los líderes de opinión. Buena parte de ellas, además, pueden ser caracterizadas como macro-influencers ( $\geq 100.000-\leq 500.000$ ): @CampAbortoLegal, @ soyingridbeck, @FundHuesped, @nbg, @porquetendencia, @PorAbortoLegal y @osvaldobazan. Dos resultan micro-influencers ( $\leq 100.000)$ : @claudiapineiro y@aministiaar. La cuenta@queridasok es seguida por pocos/as usuarios/as. 
Figura 1. Cuentas con más interacciones

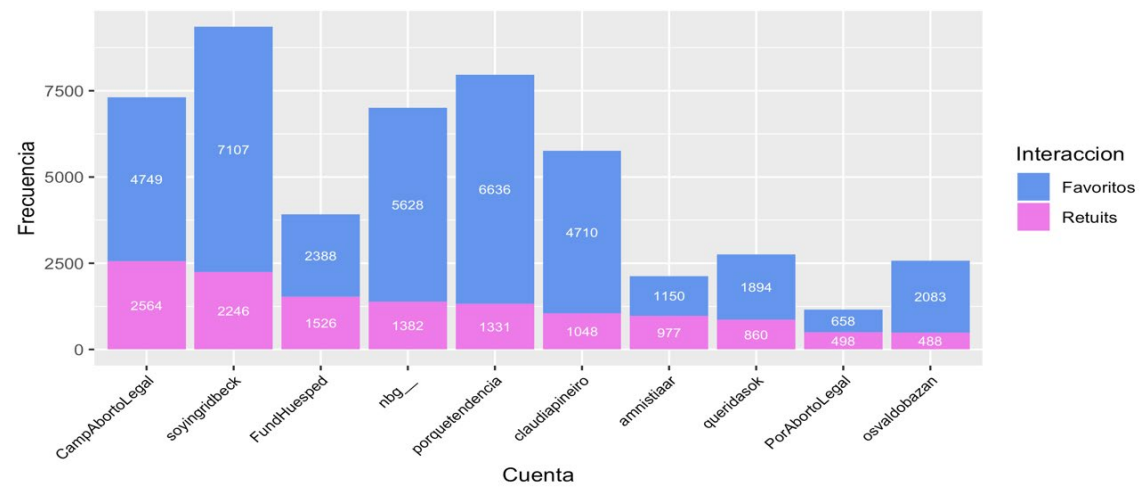

Fuente: elaboración propia.

En la Figura 1 se observa que la cuenta que logró mayor nivel de engagement pertenece a una periodista feminista (@soyingridbeck). Esta cuenta sumada a la de otros/otras dos periodistas (@nbg_, @osvaldobazan)y una escritora (@claudiapineiro) alcanzan un total de 24.692 interacciones. Por su parte, las cuentas institucionales (@CampAbortoLegal, @FundHuesped, @PorAbortoLegal, @aministiaar, @queridasok) se ubican por detrás de las cuentas personales con 17.264 interacciones. En el gráfico también aparece una cuenta que corresponde a una sección de Twitter Argentina (@porquetendencia). No se visualiza ningún usuario fake.

Por su parte, la Campaña fue la que produjo la publicación (individual) que logró más interacciones (Figura 2). Fue retuiteada 1.781 veces y recibió 3.266 favoritos. En todos esos posteos se llamaba la atención al sistema político para que tratara y aprobara en el Congreso la ley de interrupción voluntaria del embarazo. Al poseer conocimiento especializado sobre la problemática, la Campaña aparece ante los ojos de los/las usuarios/as con autoridad (Lin et al., 2018). Fue esa legitimidad la que la convirtió en referente de la conversación virtual. 
Figura 2. Publicación con más retuits
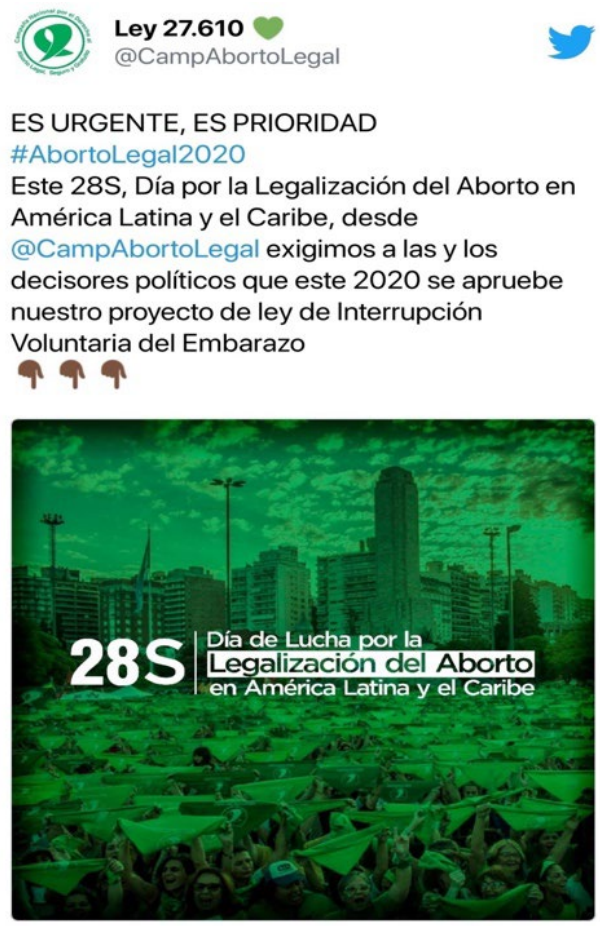

Fuente: Twitter.

El tuit con más interacciones (Figura 2) es una publicación motivacional que llama a la participación social (McDuffie \& Ames, 2021). De esta publicación se pueden inferir tres usos que le ha dado la Campaña a la etiqueta \#28S: divulgación de la convocatoria, organización de la acción y presión a los tomadores de decisión. En los tres casos es posible advertir la finalidad de su comunicación: difundir información que interpele al sistema político $\mathrm{y}$ a otros actores que puedan convertirse en potenciales aliados.

Valga agregar que la etiqueta es utilizada como un soporte de las acciones de la Campaña. Es una estrategia que suelen seguir algunos colectivos feministas (Sánchez-Duarte \& Fernández-Romero, 2017). 


\subsection{Oportunidades y narrativas}

Durante la campaña electoral de 2019, el candidato del Frente de Todos aseguró que de convertirse en Presidente trabajaría por la despenalización del aborto. Ya en 2018, Alberto Fernández se había pronunciado públicamente en su cuenta de Twitter sobre el tema:

Figura 3. Tuit de Alberto Fernández

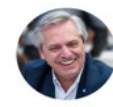

Alberto Fernández

@alferdez

La punición del aborto solo ha servido para desamparar a las mujeres pobres que no pueden recurrir a la asistencia pública para interrumpir un embarazo. El aborto no es una cuestión moral o religiosa. Es una cuestión de la salud pública. Es hora de despenalizarlo! \#AbortoLegalYa

19 Feb $2018 \cdot 22: 47$

Fuente: Twitter.

Pocos meses después de llegar al poder, en oportunidad de su discurso en la apertura del $138 .^{\circ}$ período de sesiones ordinarias del Congreso, aseguró: «[...] dentro de los próximos diez días, presentaré un proyecto de Ley de Interrupción Voluntaria del Embarazo que legalice el aborto en el tiempo inicial del embarazo y permita a las mujeres acceder al sistema de salud cuando toman la decisión de abortar» ${ }^{4}$.

La llegada de la pandemia de la COVID-19, a mediados de marzo de 2020, pospuso los planes del Ejecutivo. Todas las acciones del gobierno se concentraron, durante largos meses, en atender la crisis sanitaria y económica. No hubo otra prioridad más que la de hacer frente a una acontecimiento extraordinario, inédito e incierto.

Con un decreto presidencial de aislamiento social, preventivo y obligatorio (ASPO), la Campaña continuó su militancia desde la virtualidad.

4. Fuente: https://bit.ly/3awAPk7 Consulta: febrero de 2021. 
A pesar del contexto pandémico, la estructura de oportunidad política (EOP) (Tarrow, 2012) ofrecía incentivos para la acción colectiva. No sólo el Presidente se había pronunciado ya sobre el tema sino que el propio estrés de la sanidad pública abría la posibilidad para que la Campaña instalara la narrativa de que la legalización del aborto sería una herramienta muy importante que descomprimiría la ocupación de los hospitales a causa de internaciones por complicaciones de abortos clandestinos.

Con una EOP permeable, durante los días previos al 28S, la Campaña emitió dos comunicados y alentó la participación al «pañuelazo virtual». En el documento titulado «Es urgente, es prioridad», que fue publicado en los medios de comunicación, la Campaña reclamaba que su proyecto de Ley de Interrupción Voluntaria del Embarazo, impulsado por más de setecientas organizaciones de diversa índole, se aprobara de manera urgente. Apelaba, en ese sentido, a que los poderes públicos del Estado argentino lo reglamentaran como un derecho efectivo.

En el segundo documento titulado «Un grito recorre América Latina y el Caribe. 28 de septiembre, día de lucha por el aborto legal» insistía con el reclamo, pero le agregaba la dimensión regional: «La Campaña 28 de Septiembre se propone exigir las condiciones para el aborto seguro, con calidad de atención y los cambios jurídicos que otorguen plenos derechos en cada uno de los países de América Latina y el Caribe» ${ }^{5}$.

En ambos documentos se hacía énfasis, además, en la lucha del feminismo por una ley que representaba una cuestión de salud pública y en la exigencia de reconocer el derecho de las mujeres a decidir sobre su propio cuerpo. En síntesis, la demanda de una política pública de salud integral que proporcionara mayor autonomía y libertad a las mujeres.

El «pañuelazo virtual», por su parte, superó las expectativas de las organizadoras. A las 18 del 28S, miles de activistas de toda Argentina coparon las redes sociales. Zoom, la plataforma de videoconferencias, sobrepasó el cupo de los mil asistentes. Hubo más de seis mil seguidores en la transmisión de YouTube y otros dos mil en Facebook. Fue, además, tendencia en Twitter Argentina ${ }^{6}$.

5. Fuente: http://bit.ly/37pQgst Consulta: enero de 2021.

6. Fuente: https://bit.ly/2ZGKkqV Consulta: febrero de 2021.

Feminismo/s 39, January 2022, 13-37 
Se ha aplicado un tratamiento automático de textos al volumen de tuits originales que se han identificado $(n=8.200)$ para determinar si las narrativas que producen los/las usuarios/as están alineadas a las consignas de la Campaña. La frecuencia de términos utilizados en sus publicaciones nos permite dar cuenta de los principales tópicos de la conversación digital.

Figura 4. Palabras más usadas en los tuits

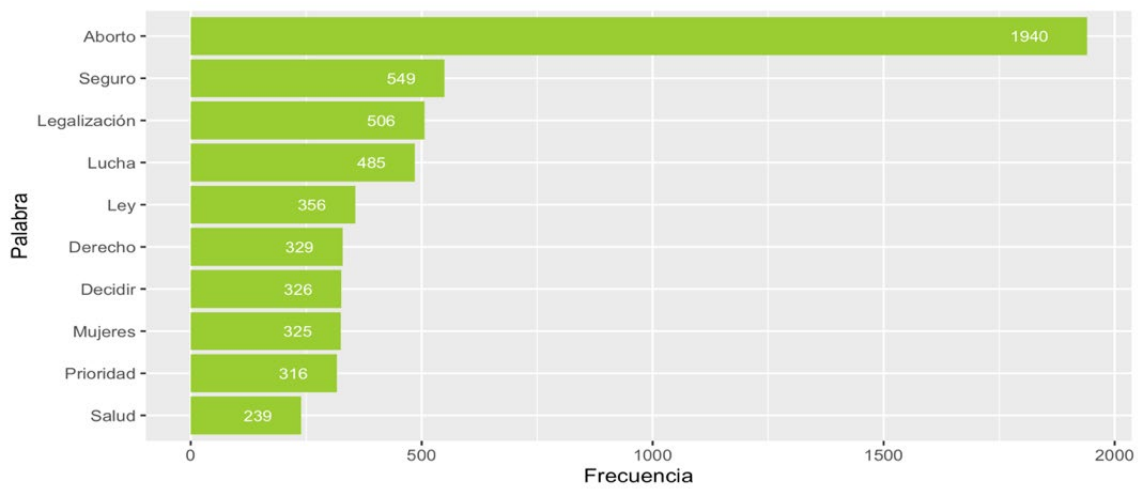

Fuente: elaboración propia.

La reiteración de un conjunto de palabras (Figura 4) nos permite afirmar que en sus publicaciones, los/las usuarios han retomado las narrativas activadas por la Campaña en sus documentos públicos. Allí reside el éxito de la campaña tecnopolítica que desarrolló desde 2018 cuando además de hegemonizar la discusión sobre el aborto y crear un conjunto de marcos cognitivos sobre la problemática, organizó la movilización en las calles y motivó un movimiento de derechos reproductivos en gran parte de América Latina (Acosta, 2018).

La acción del \#28S impulsada en 2020 por la Campaña pudo haber sido, además, un factor de presión para que el presidente Fernández anunciara, poco después, el envío al Congreso de un proyecto que legalizaría el aborto y garantizaría que el sistema de salud permitiera su realización en condiciones sanitarias a fin de asegurar la salud y la vida de las personas gestantes. La propuesta del Ejecutivo recogía los consensos del debate legislativo de 2018. 
La Campaña celebró la presentación del proyecto oficial, que había sido prometido durante la campaña electoral de 2019, y reconoció coincidencias «muy significativas» con él al tiempo que mostró reparos respecto de la mención de la Objeción de Conciencia del personal médico a cargo de prestar el servicio o de proveer la práctica, los plazos para acceder a ella y la inclusión del derecho al aborto como contenido curricular de la Educación Sexual Integral (ESI).

\section{DISCUSIÓN Y CONCLUSIONES}

El análisis de contenido realizado valida las hipótesis planteadas. Esto es: 1) en la etiqueta predominan usuarios/as que participan a título personal y, en general, tienen pocos seguidores; 2) la conversación digital es guiada por líderes de opinión (periodistas) que son quienes producen mayores niveles de interacción y 3) las narrativas que producen los/las usuarios/as están alineadas a las consignas trazadas por la Campaña.

La etiqueta \#28S ha sido utilizada para visibilizar la causa por el aborto y reunir apoyo. No obstante, tal como han observado algunas investigaciones, esa estrategia es susceptible de conllevar un doble problema. Por un lado, la discusión puede reducirse a una simple acción de retuiteo de publicaciones que termina encapsulándose entre cuentas afines. En lugar de propiciar el debate, se favorece el simple agrupamiento de cuentas que opinan parecido y se pierde la posibilidad de promover colectividades fuertes (Caro Castaño, 2015; Fotopoulou, 2014; Iranzo-Cabrera, 2020). Por otro lado, la mera participación de usuarios/as en los hashtags no alienta necesariamente algún tipo de acción y conlleva el riesgo de estancarse en una experiencia cómoda y privada (Dahlgren, 2018).

A pesar de estas advertencias, creemos que la Campaña ha sorteado con éxito esa potencial limitación y ha logrado crear identidades sólidas a través de sus dinámicas digitales, especialmente entre las mujeres más jóvenes (Acosta, 2020). Sin ir más lejos, en el caso del \#28S, y por la contingencia de la pandemia, la acción que promovió desde las redes sociales denominada «pañuelazo verde» fue un éxito del que participaron miles de mujeres en todo el país. 
La comunicación que se genera en las plataformas digitales sirve no sólo para visibilizar la necesidad de ampliar derechos y materializar las demandas sino también para establecer relaciones estratégicas con potenciales aliados tales como tomadores de decisiones, medios de comunicación y líderes de opinión. Hemos visto que la acción comunicativa (Habermas, 2010) que la Campaña ha desarrollado en las redes sociales a través de la etiqueta ha buscado influir en la agenda del sistema político, a partir de una EOP que ofreció incentivos para la acción colectiva.

Esta investigación no incluye el análisis de las comunidades virtuales que participaron del hashtag. Tampoco los hashtags antagonistas utilizados por los contramovimientos. Una agenda investigativa futura podría contemplar ambas dimensiones. La primera serviría para caracterizar a los clusters que intervienen en la discusión y dar cuenta de los arquetipos que se configuran; la segunda sería fundamental para conocer en detalle el uso que hacen de las plataformas digitales los colectivos que buscan posicionarse contra la agenda feminista. Esto último resulta crucial. En la mayoría de los países de América Latina no se han producido avances constantes en los procesos que se venían dando respecto de los derechos sexuales. En algunos casos se presentan importantes estancamientos, persisten sesgos anclados en códigos culturales y faltan más políticas públicas (Montenegro et al., 2020). Paralelamente, se experimenta el avance de movimientos backlash (Biroli \& Caminotti, 2020; Bosia et al., 2020). La intervención de usuarios/as de etiquetas relacionadas con vindicaciones conservadoras coloca en el centro de la cuestión a estudiar las tensiones y desafíos que se le plantean al feminismo en la región.

\section{REFERENCIAS BIBLIOGRÁFICAS}

Accossatto, R., \& Sendra, M. (2018). Movimientos feministas en la era digital. Las estrategias comunicacionales del movimiento Ni Una Menos. Encuentros: Revista de Ciencias Humanas, Teoría Social y Pensamiento Crítico, 8, 117-136. https://bit.ly/3s3mPUW

Acosta, M. (2018). Ciberactivismo feminista. La lucha de las mujeres por la despenalización del aborto en Argentina. Sphera Publica, 2(18), 2-20. https:// bit.ly/36QysGl

Acosta, M. (2020). Activismo feminista en Instagram. El caso de la campaña nacional por el derecho al aborto legal seguro y gratuito en Argentina.

Feminismo/s 39, January 2022, 13-37 
Perspectivas de la Comunicación, 13(1), 29-46. https://doi.org/10.4067/ S0718-48672020000100029

Alcaraz, F. (2018). ¿Que sea ley!. La lucha de los feminismos por el aborto legal. Marea.

Arias Rodríguez, A. (2017). La cimentación social del concepto mujer en la red social Facebook. Revista de Investigación Educativa, 35(1), 181-195. http:// bit.ly/3pvcBes

Bardin, L. (1986). El análisis de contenido. Akal.

Biroli, F., \& Caminotti, M. (2020). The Conservative Backlash against Gender in Latin America. Politics \& Gender, 16(1). https://doi.org/10.1017/ S1743923X20000045

Bosia, M., McEvoy, S., \& Rahman, M. (2020). The Oxford Handbook of Global LGBT and Sexual Diversity Politics. Oxford Handbooks.

Calvo, E. \& Aruguete, N. (2020). Fakenews, burbujas, trolls y otros encantos: cómo funcionan (para bien y para mal) las redes sociales. Siglo XXI.

Caro Castaño, L. (2015). Construir y comunicar un «nosotras» feminista desde los medios sociales. Una reflexión acerca del «feminismo del hashtag.» Commons, 4(2), 124-154. https://bit.ly/3026GGs

Castells, M. (1998). La Era de la información: economía, sociedad y cultura. Alianza.

Castells, M. (2009). Comunicación y poder. Alianza.

Castells, M. (2013). Redes de indignación y esperanza: los movimientos sociales en la era de Internet. Alianza.

Ceron, A., Curini, L., Iacus, S., \& Porro, G. (2013). Every tweet counts? How sentiment analysis of social media can improve our knowledge of citizens' political preferences with an application to Italy and France. New Media $\mathcal{E}$ Society, 16(2), 340-358. https://doi.org/10.1177/1461444813480466

Clark, R. (2016). Hope in a hashtag»: the discursive activism of \#WhylStayed. Feminist Media Studies, 16(5), 788-804. https://doi.org/10.1080/14680777.2 016.1138235

Congosto, M., Deltell Escolar, L., Claes, F., \& Osteso, J. M. (2013). Análisis de la audiencia social por medio de Twitter. Caso de estudio: los premios Goya 2013. Revista ICONO14. Revista Científica de Comunicación y Tecnologías Emergentes, 11(2), 53-82. https://doi.org/10.7195/ril4.v1li2.577

Cresswell, J. (2015). A Concise Introduction to Mixed Methods Research. Sage. 
Dahlgren, P. (2018). La participación en línea en la esfera pública. Las ambigüedades del afecto. InMediaciones de La Comunicación, 13(1), 25-47. https:// bit.ly/3kZKbJS

della Porta, D. (2008). Prefacio. En A. Grimson \& S. Pereyra (Eds.). Conflictos globales, voces locales. Movilización y activismos en clave transnacional (pp.1116). Prometeo.

della Porta, D., \& Diani, M. (2015). Los movimientos sociales. UCM.

Deltell, L., Congosto, M., Claes, F., \& Osteso, J. M. (2013). Identificación y análisis de los líderes de opinión en Twitter en torno a Hugo Chávez (Identification and analysis of opinion leaders on Twitter around Hugo Chávez). Revista Latina de Comunicación Social, 68, 696-718. https://doi. org/10.4185/RLCS-2013-997

Elizalde, S. \& Mateo, N. (2018). Las jóvenes: entre la «marea verde» y la decisión de abortar. Salud colectiva, 14 (3), 433-446. https://doi.org/10.18294/ sc. 2018.2026

Fotopoulou, A. (2014). Digital and networked by default? Women's organisations and the social imaginary of networked feminism. New Media and Society, 18(6), 1-17. https://doi.org/10.1177/1461444814552264

Fraser, N. (2008). La justicia social en la era de la política de la identidad: redistribución, reconocimiento y participación. Revista de Trabajo, 4(6), 83-99. http://bit.ly/2NAYSWe

Fraser, N. (2012). La política feminista en la era del reconocimiento: un enfoque bidimensional de la justicia de género. Arenal, 19(2), 267-286. https://bit. ly/2NyZU4W

Georgakopoulou, A. (2017). Sharing the moment as small stories: The interplay between practices $\&$ affordances in the social media-curation of lives. Narrative Inquiry, 27, 311-333. https://doi.org/10.1075/ni.27.2.06geo

Gerbaudo, P. (2012). Tweets and the Streets: Social Media and Contemporary Activism. Pluto Books.

Gong, R. (2014). Indignation, inspiration, and interaction on the Internet: Emotion work online in the anti-trafficking movement. Journal of Technology in Human Services, 33(1), 87-103. https://doi.org/10.1080/15228835.2014.99 8988

Gundecha, P., \& Liu, H. (2014). Mining Social Media: A Brief Introduction. Informs. Tutorials in Operations Research, 1-17. http://dx.doi.org/10.1287/ educ.1120.0105 
Gutiérrez, M.A. (2014). Aborto y ciudadanía: la Campaña Nacional por el Derecho al Aborto legal, seguro y gratuito en Argentina. Espacios Políticos. http:// espaciospoliticos.org/aborto-y-ciudadania-la-campana-nacional-por-el-derecho-al-aborto-legal-seguro-y-gratuito-en-argentina/

Habermas, J. (1998). Facticidad y validez. Trotta.

Habermas, J. (2010). Teoría de la acción comunicativa. Trotta.

Hernández Sampieri, R., Fernández Collado, C., \& Baptista, P. (1991). Metodología de la investigación. McGraw-Hill.

Hwang, S. (2008). Utilizing qualitative data analysis software: A review of ATLAS.ti. Social Science Computer Review, 26(4), 519-527. https://doi. org/10.1177/0894439307312485

Iranzo-Cabrera, M. (2020). \#lasperiodistasparamos, gestación de una conciencia profesional feminista. El Profesional de La Información, 29(2). https://doi. org/10.3145/epi.2020.mar.22

Konnelly, A. (2015). \#Activism: Identity, Affiliation, and Political DiscourseMaking on Twitter. The Arbutus Review, 6(1), 1-16. https://doi.org/10.18357/ ar.konnellya.612015

Larrondo, A., Morales i Gras, J. \& Orbegozo Terradillos, J. (2019). Feminist hashtag activism in Spain: measuring the degree of politicisation of online discourse on \#YoSíTeCreo, \#HermanaYoSíTeCreo, \#Cuéntalo y \#NoEstásSola. Communication \& Society, 32 (4), 207-221. https://doi. org/10.15581/003.32.4.207-221

Lin, H.-C., Bruning, P., \& Swarna, H. (2018). Using online opinion leaders to promote the hedonic and utilitarian value of products and services. Business Horizons, 61, 431-442. https://bit.ly/3HPK1P5

Lucaccini, M., Zaidan, L. \& Pecheny, M. (2019), Qué nos dice el debate sobre aborto en 2018 sobre la clase política y el espacio público en la Argentina. En D. Maffia, P. Gómez \& A. Moreno (Comps.), Miradas feministas sobre los derechos (pp. 245-263). Editorial Jusbaires.

McAdam, D., Tarrow, S., \& Tilly, C. (2005). Dinámica de la contienda política. Hacer.

McDuffie, K., \& Ames, M. (2021). Archiving affect and activism: Hashtag feminism and structures of feeling in Women's March tweets. First Monday, 26(2). https://doi.org/10.5210/fm.v26i2.10317 
Montenegro, M., Herrera Montenegro, L., \& Torres-Lista, V. (2020). Los Derechos de las personas LGBTIQ+, agenda de género y las políticas de igualdad. Encuentros, 11, 9-23. https://doi.org/10.5281/zenodo.3687275

Natalucci, A., \& Rey, J. (2018). ¿Una nueva oleada feminista?. Agendas de género, repertorios de acción y colectivos de mujeres (Argentina, 2015-2018). Revista de Estudios Políticos y Estratégicos, 6(2), 14-34. http://bit.ly/3puE3c5

Nathanson, G., \& Paz, M. (2019). Ciberfeminismos contemporáneos, entre usos y apropiaciones. Cadernos Pagu, 56, 1-29. https://doi.org/10.1590/1809444 9201900560022

Norris, P. (2002). Democratic Phoenix: Reinventing Political Activism. Cambridge University Press.

Núñez Puente, S., \& Fernández Romero, D. (2017). Narrativas transformadoras y testimonio ético las estrategias discursivas de la Plataforma Feminista 7N, Contra las Violencias Machistas. Index.Comunicación, 7(3), 269-281. http://bit.ly/3jZkpE5

Padua, J. (2000). Técnicas de investigación aplicadas a las ciencias sociales. FCE. Pérez Guadarramas, Y., Rodríguez, A., Simón, A., Hoyas, W., \& Olivas, J. (2017). Combinando patrones léxico-sintácticos y análisis de tópicos para la extracción automática de frases relevantes en textos. Procesamiento Del Lenguaje Natural, 59, 39-46. http://bit.ly/2OPKXwl

Ramírez Morales, M., \& Felitti, K. (2020). Pañuelos verdes por el aborto legal. Encartes, 3(5), 111-145. https://doi.org/10.29340/en.v3n5.132

Resina de la Fuente, J. (2010). Ciberpolítica, redes sociales y nuevas movilizaciones en España: el impacto digital en los procesos de deliberación y participación ciudadana. Mediaciones Sociales, 7, 143-164. http://bit.ly/3aw7HJO Rosales, M. (2018). Ciberactivismo: praxis feminista y visibilidad política en \#NiUnaMenos. Pleyade, 22. http://dx.doi.org/10.4067/ S0719-36962018000200063

Sábada, I., \& Barranquero, A. (2019). El ciberfeminismo como objeto de investigación. Athenea Digital, 19(1), 1-24. https://bit.ly/3FAPd7u

Sampedro Blanco, V. \& Resina de la Fuente, J. (2010). Opinión pública y democracia deliberativa en la Sociedad Red. Ayer, 80 (4), 139-162.

Sánchez-Duarte, J.-M., \& Fernández-Romero, D. (2017). Subactivismo feminista y repertorios de acción colectiva digitales: prácticas ciberfeministas en Twitter. El Profesional de La Información, 26(5), 894-902. https://doi. org/10.3145/epi.2017.sep.11 
Silge, J., \& Robinson, D. (2017). Text mining with R. A tidy approach. O'Reilly Media.

Sola-Morales, S., \& Sabariego, J. (2020). Tecnopolítica, recientes movimientos sociales globales e Internet. Una década de protestas ciudadanas. Teknocultura, 17(2), 194-203. https://doi.org/10.5209/tekn.66241

Tarrow, S. (2005). The New Transnational Activism. Cambridge University Press. Tarrow, S. (2012). El poder en movimiento. Los movimientos sociales, la acción colectiva y la política. Alianza.

Tilly, C. (2000). Acción colectiva. Apuntes de investigación del CECyP, 6, 9-32.

Tilly, C. (2005). Los movimientos sociales entran en el siglo veintiuno. Política y Sociedad, 42(2), 11-35. http://bit.ly/2Mlah0U

Tilly, C. (2010). Los movimientos sociales, 1768-2009: Desde sus orígenes a Facebook. Akal.

Toret, J. (2013). Tecnopolítica: la potencia de las multitudes conectadas. El sistema red 15M, un nuevo paradigma de la política distribuida. IN3 Working Paper Series, 1-178. https://doi.org/10.7238/in3wps.v0i0.1878

Toret, J. (2015). Tecnopolítica y 15M. La potencia de las multitudes conectadas. Un estudio sobre la gestación y explosión del 15M. UOC.

Treré, E. (2016). Distorsiones tecno políticas: represión y resistencia algorítmica del activismo ciudadano en la era del big data. Tripodos, 39, 35-51. http:// bit.ly/3pt2Tcx

Valles, M. (2000). Técnicas cualitativas de investigación social. Editorial Síntesis. Vallès, J. \& Martí, S. (2016). Ciencia Política. Un manual. Ariel.

Van-Laer, J., \& Van-Aelst, P. (2010). Internet and social movement action repertoires. Information, Communication \& Society, 13(8), 1146-1171. http://dx.doi. org/10.1080/13691181003628307

Van Dijck, J. (2016). La cultura de la conectividad. Una historia crítica de las redes sociales. Siglo XXI.

Yang, G. (2016). Narrative Agency in Hashtag Activism: The Case of \#BlackLivesMatter. Media and Communication, 4(4), 13-17. https://doi. org/10.17645/mac.v4i4.692

Zafarani, R., Ali Abbasi, M., \& Liu, H. (2014). Social Media Mining: an Introduction. Cambridge University Press. https://dx.doi.org/10.1017/cbo9781139088510 Zappavigna, M. (2015). Searchable talk: the linguistic functions of hashtags. Social Semiotics, 25, 274-291. https://doi.org/10.1080/10350330.2014.996948 